\title{
DNA self-assembly scaled up
}

DNA can be designed to self-assemble into target shapes, but the size and quantity of objects that can be prepared have been limited. Methods to overcome these problems have now been found. SEE LETTERS P.67, P.72, P.78 \& P.84

\section{FEI ZHANG \& HAO YAN}

elf-assembly processes occur in nature

$\circlearrowleft$ in various forms, from molecular-level protein folding and the formation of lipid bilayers to the establishment of Earth's entire biological system ${ }^{1}$. Scientists have long aspired to construct artificial objects using self-assembly to reach the dimensions and complexity of cells or organelles, with the aim of building synthetic cellular machines for research, engineering and medical applications. Four papers $^{2-5}$ in this issue address this goal by reporting methods for scaling up the sizes and production of self-assembling, designer nanostructures made from DNA.

The biopolymers DNA, RNA and proteins have all been used as building blocks for the assembly of designer nanoscale architectures, to engineer bioinspired or biomimetic systems that can communicate with each other ${ }^{6}$ and to regulate the functions of living organisms ${ }^{7}$. DNA is the most useful nanoscale building block because it has several advantages especially its programmability, which derives from the predictable and stable pairs that form between bases on complementary DNA strands. Moreover, DNA is structurally stable, the geometrical features of its double helix have been well studied, and it is compatible with other biological molecules, which should allow the construction of 'hetero-biomaterials' that have complex functions. Various DNA self-assembly methods (see ref. 8, for example) have been developed for constructing synthetic architectures that exhibit great geometrical complexity and nanoscale accuracy.

One of the milestones in DNA nanotechnology was the invention of DNA origami ${ }^{9}$. In this technique, long, single-stranded DNA is folded into target shapes with the help of hundreds of short DNA strands called staples. The staple strands are designed to be complementary to particular regions of the long DNA, and thereby guide the folding process. A wide variety of 2D and 3D nano-objects has been made using this technique. Many of these are fully addressable ${ }^{10}$; that is, they can be modified at selected positions, as needed for future applications. However, the size of individual DNA origami nanostructures is limited by the length of the scaffold DNA from which they are built. For
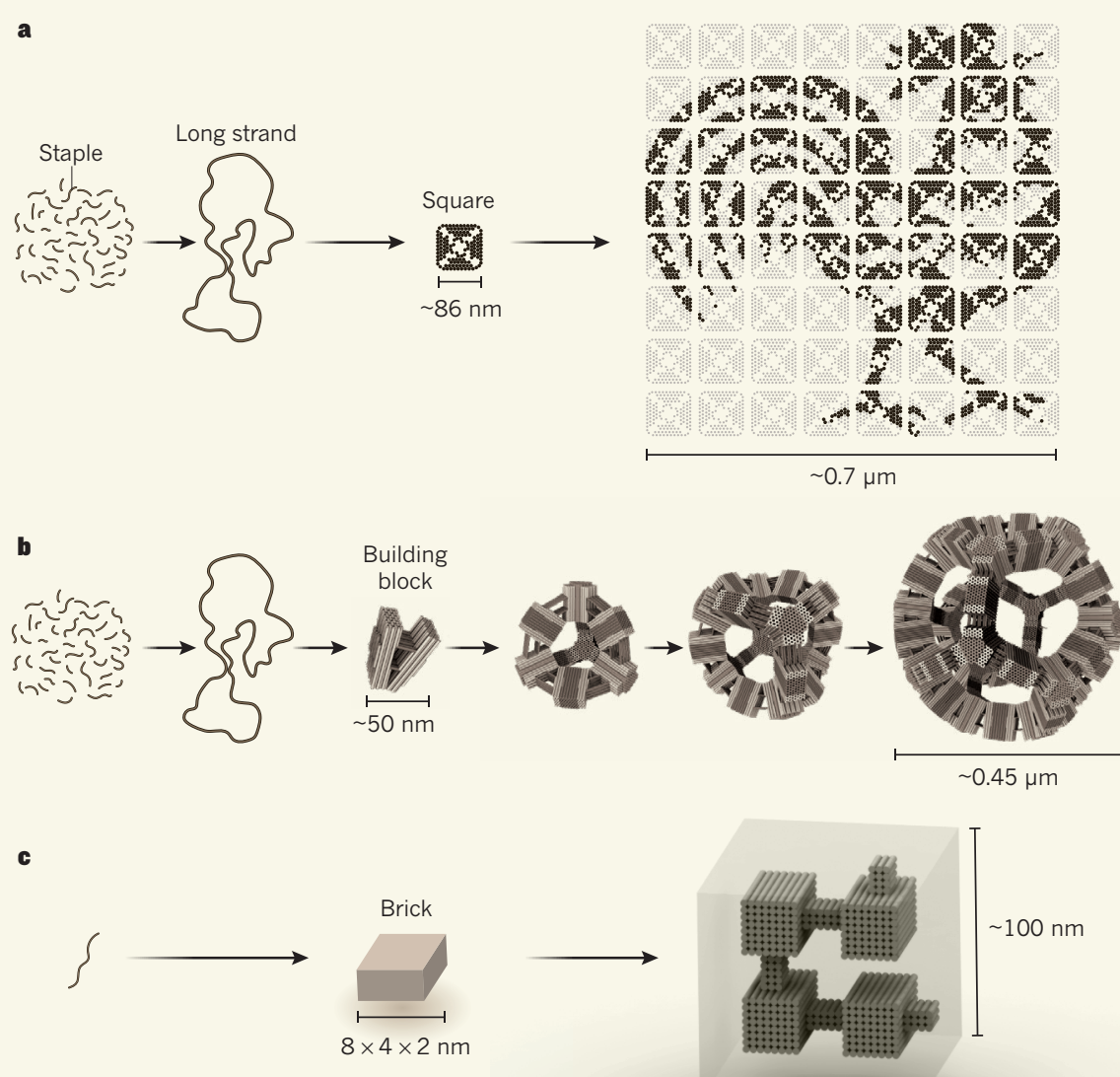

Figure 1 | Methods for making micrometre-scale DNA objects. a, Tikhomirov et al. ${ }^{2}$ used DNA origami - a technique in which small DNA strands (staples) guide the self-assembly of a longer strand to prepare squares. These squares were assembled into $8 \times 8$ arrays that had customized surface patterns. b, Wagenbauer et al. ${ }^{3}$ prepared a 3D, V-shaped building block using DNA origami, and assembled this hierarchically into polyhedra. c, Ong et al. ${ }^{4}$ built on a method known as single-stranded tile assembly to prepare DNA bricks that form one strand that contains 52 nucleotides. Each strand contains four binding domains that enable the bricks to assemble into larger constructions. Thousands of bricks can be programmed to self-assemble into a cuboid that contains a complex cavity.

example, one widely used scaffold is a genomic DNA approximately 7,200 nucleotides long', which folds into origami structures no more than 100 nanometres in diameter ${ }^{9,10}$.

Another important design strategy in DNA nanotechnology is single-stranded tile (SST) assembly ${ }^{11}$, in which SSTs - nanometre-scale $2 \mathrm{D}$ rectangles or $3 \mathrm{D}$ bricks, formed from single-stranded DNA — are designed to interlock with each other through the formation of DNA duplexes at their interfaces. Collections of SSTs are used to form 2D sheets or 3D blocks that can be selectively 'sculpted' to create different patterns and shapes, simply by including or omitting specific SSTs ${ }^{12}$. But the sizes of the DNA structures produced in this way are generally comparable to the sizes of origami nanostructures; larger structures have been prepared only in low yields. The papers reported in this issue build on the SST and origami strategies to 
make micrometre-sized structures, and to scale up the amounts that can be produced.

Tikhomirov et al. ${ }^{2}$ (page 67) used square DNA origami decorated with surface patterns (formed by DNA strands that extend from the origami surface) as building units to create 2D DNA origami arrays up to about half a micrometre across (Fig. 1a). The square origami join together through the formation of short DNA duplexes at their interfaces. To program the interactions between the square origami, the authors developed a fractal method in which local assembly rules were used recursively in a hierarchical, multi-step process that assembles increasingly large arrays of the square origami. Tikhomirov and colleagues also produced design software called FracTile Compiler, which will enable non-experts to devise DNA sequences and experimental procedures to make large DNA patterns. The authors validated this automated design process by using it to make several DNA 'pictures', including the Mona Lisa, a rooster and a chess-game pattern (see Fig. 3 of the paper ${ }^{2}$ ).

Wagenbauer et al. ${ }^{3}$ (page 78 ) have made 3D DNA origami structures at sizes up to the micrometre scale, using another hierarchical self-assembly approach (Fig. 1b). They used a V-shaped DNA origami object as the basic building block, in which the angle of the $\mathrm{V}$ could be altered. By controlling the geometry and interactions between the building blocks, higher-order assemblies can be constructed. The authors demonstrated the capabilities of their method by constructing micrometrelong tubes (similar in size to some bacteria) out of stacked planar rings up to $350 \mathrm{~nm}$ in diameter, and three types of polyhedron up to $450 \mathrm{~nm}$ in diameter.

Ong et al. ${ }^{4}$ (page 72 ) report a method that allows 3D SST DNA constructs to be made at the micrometre scale (Fig. 1c). By extending the principles of first-generation SST systems, the authors designed a brick-shaped DNA building block composed of 52 nucleotides, which contains four 13-nucleotide binding domains. These domains enable the bricks to assemble into larger constructions. Compared with the first-generation bricks (which contained four binding domains, each composed of eight nucleotides), the longer binding domains of the DNA bricks provide better yields and stabilities for large assembled structures. The authors developed software called Nanobricks to design the brick strands needed to make target $3 \mathrm{D}$ objects, and used it to plan the synthesis of a set of different complex architectures (see Fig. 3 of the paper ${ }^{4}$ ).

Praetorius et al. ${ }^{5}$ (who belong to the same research group as Wagenbauer and colleagues; page 84) report biotechnology that should greatly reduce the cost of the hundreds of staple strands that are usually used to make DNA origami. They use viruses known as bacteriophages to produce single-stranded precursor DNA that contains hundreds of staple-strand sequences. These sequences are separated by a 'DNAzyme' sequence that cleaves itself; the cleavage products then self-assemble into designated DNA origami shapes. Remarkably, the authors' method reduces the cost of the folded DNA origami structures from about US $\$ 200$ per milligram to around 20 cents. This strategy will enable scalable and efficient mass production of DNA origami and SST structures, thus enabling large-scale applications, such as therapeutics, drug-delivery systems and nanoelectronic devices.

The papers also offer solutions for longstanding challenges in the field of biomolecular engineering, providing low-cost methods for fabricating self-assembled structures from smaller building blocks, at sizes that can be integrated into objects made using complementary 'top-down' techniques (those that carve structures out of bulk material). Furthermore, the reported DNA structures are large enough to enable the production of devices that interact with cells for therapeutic applications, or to make sophisticated molecular machines and assembly lines that make synthetic polymers or program cell-cell interactions. Such self-assembled structures might even be used in synthetic organelles to create systems that sense, monitor and regulate biological processes in living cells.

Fei Zhang and Hao Yan are at the Biodesign Center for Molecular Design and Biomimetics, Biodesign Institute, and at the School of Molecular Sciences, Arizona State University, Tempe, Arizona 85287, USA.

e-mail:hao.yan@asu.edu

1. Whitesides, G. M. \& Grzybowski, B. Science $\mathbf{2 9 5}$ 2418-2421 (2002).

2. Tikhomirov, G. et al. Nature 552, 67-71 (2017)

3. Wagenbauer, K. F. et al. Nature 552, 78-83 (2017).

4. Ong, L. L. et al. Nature 552, 72-77 (2017).

5. Praetorius, F. et al. Nature 552, 84-87 (2017).

6. Chen, Y.-J., Groves, B., Muscat, R. A. \& Seelig, G. Nature Nanotechnol. 10, 748-760 (2015).

7. Li, J., Green, A. A., Yan, H. \& Fan, C. Nature Chem. $\mathbf{9}$, 1056-1067 (2017)

8. Zheng, J. et al. Nature 461, 74-77 (2009).

9. Rothemund, P. W. K. Nature 440, 297-302 (2006).

10.Douglas, S. M. et al. Nature 459, 414-418 (2009).

11.Wei, B., Dai, M. \& Yin, P. Nature 485, 623-626 (2012)

12.Ke, Y., Ong, L. L., Shih, W. M. \& Yin, P. Science 338,

$1177-1183$ (2012)

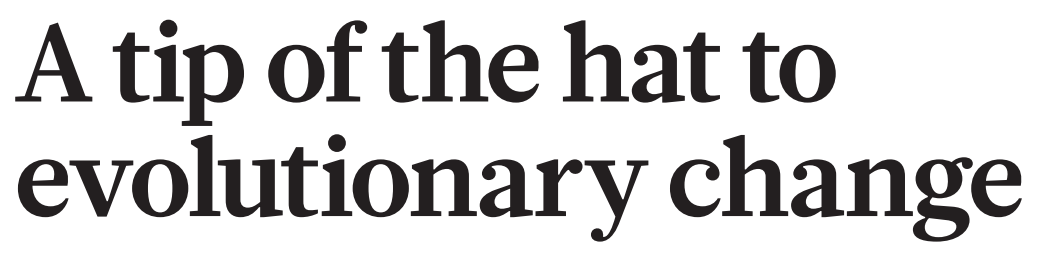

The relative roles of biological and environmental factors in driving evolutionary change have been unclear. Now fossil analysis shows that their action depends on where an animal group is in its evolutionary trajectory. SEE LETTER P.92

\section{CHARLES R. MARSHALL}

A ssessing the relative importance of biological interactions (biotic factors) and changes in the physical environment (abiotic factors) in driving evolution has proved difficult, partly owing to the lack of high-quality data sets that combine both fossil remains and associated ancient environmental data. The few studies that have quantified the relative contributions of these two drivers describe a complex relationship ${ }^{1}$. However, on page 92, Žliobaitè et al. ${ }^{2}$ report an unexpectedly simple pattern of driver action in peak evolutionary success.

Between a species originating and becoming extinct, its evolutionary success can be measured in a number of ways, such as the extent of its geographical range. Such metrics often form a 'hat-shaped' curve, with a rise towards a central peak, followed by a decline to extinction. Why this pattern occurs so often and the degree to which biotic and abiotic factors influence this trajectory is a matter of debate.

To assess the relative role of biotic factors, such as competition between organisms, and abiotic factors in evolutionary trajectories, Žliobaite and colleagues analysed the fossil record of large herbivorous mammals. This grouping offers several advantages for this type of analysis. For instance, the authors could solve the problem of finding consistent regional ancient environmental data because the height of these mammals' teeth correlates strongly with characteristics of their environment, including precipitation levels ${ }^{3}$ and the amount of plant material in the ecosystem ${ }^{4}$. Without this measure, the authors would have had to rely on standard global measurements of environmental change, an approach that can mask substantial regional-level variation.

Another advantage is that these animals all share a similar ecological niche, so the authors could use the average number of genera (groups of closely related species) per locality to measure competition intensity, a biotic factor that is otherwise difficult to quantify. In addition, the fossil record of large mammalian herbivores is sufficiently rich that the proportion of localities in which 